\title{
Two new Perseus arm supernova remnants discovered in the Canadian Galactic Plane Survey
}

\author{
R. Kothes ${ }^{1,2}$, B. Uyanıker ${ }^{1,3}$, and R. I. Reid ${ }^{1}$ \\ ${ }^{1}$ National Research Council of Canada, Herzberg Institute of Astrophysics, Dominion Radio Astrophysical Observatory, PO Box 248, \\ Penticton, British Columbia, V2A 6J9, Canada \\ 2 Department of Physics and Astronomy, The University of Calgary, 2500 University Dr. NW, Calgary, AB, T2N 1N4, Canada \\ 3 Max-Planck-Institut für Radioastronomie, Auf dem Hügel 69, 53121 Bonn, Germany \\ e-mail: roland.kothes@nrc-cnrc.gc.ca
}

Received 15 July 2005 / Accepted 24 August 2005

\section{ABSTRACT}

We report the discovery of two new second-quadrant supernova remnants, G96.0+2.0 and G113.0+0.2, in the data of the Canadian Galactic Plane Survey. The two SNRs are residents of the Perseus spiral arm at distances of $4.0 \mathrm{kpc}$ and $3.1 \mathrm{kpc}$, respectively. The distances were determined kinematically by associating the objects with neutral hydrogen and molecular material.

G96.0+2.0 is most likely located at the edge of a large stellar wind bubble with a systemic velocity of about $-44 \mathrm{~km} \mathrm{~s}^{-1}$. It consists of a relatively bright shell where the shock is encountering the wall of $\mathrm{HI}$ and slowly fades away towards the interior of the stellar wind bubble. The visible part of the remnant has a diameter of about $30 \mathrm{pc}$ and a radio spectral index of $\alpha \approx-0.66\left(S \sim v^{\alpha}\right)$, indicating that it is a shell-type remnant in an early stage of development. The SNR is most likely the remnant of a type $\mathrm{Ib} / \mathrm{c}$ supernova explosion.

G113.0+0.2 is located in an area of confusing thermal emission not far from the radio-bright supernova remnant Cassiopeia A. It has an unusual elongated structure consisting of a long polarized filament and a more complex head structure that is interacting with a small molecular cloud; it resides in a butterfly-shaped H I cavity, probably a stellar wind bubble. It is about $36 \mathrm{pc}$ long and $15 \mathrm{pc}$ wide at a position angle of $70^{\circ}$ with the Galactic Plane. A pulsar with a relatively low period derivative, giving it a characteristic age of 10 million years, is located close to the centre of the radio continuum emission at a Perseus arm distance. Whether the pulsar is the result of the same supernova explosion that created G113.0+0.2 or if it was left behind by an earlier supernova that also shaped the stellar wind bubble remains uncertain.

Key words. ISM: individual objects: G113.0+0.2, G96.0+2.0 - ISM: supernova remnants - ISM: bubbles - polarization

\section{Introduction}

Unbiased, high resolution, wide-field images of all major constituents of the Galactic interstellar medium (ISM) in the Outer Galaxy are now available in the Canadian Galactic Plane Survey (CGPS, Taylor et al. 2003). The CGPS data, probing the ionized, neutral, particulate, and magneto-ionic media over unprecedented spatial dynamic range, are allowing new insights into the SNR population in the Outer Galaxy. As a part of the ongoing CGPS effort to study the characteristics of supernova remnants and their environment we searched the whole area of the CGPS for new SNR candidates. Currently 231 supernova remnants have been identified in the radio band after decades of intensive search with the largest single-antenna and synthesis telescopes (Green 2004a,b). Our current view of supernova remnants (SNRs) has been shaped by the study of the radio bright objects, all of which have probably now been detected. On the other hand, the population of SNRs in the Galaxy is likely to be dominated by objects with low radio surface brightness. These could be old SNRs that are slowly fading away or young SNRs expanding inside stellar wind bubbles. The discovery of these faint objects requires a survey which combines high sensitivity with excellent angular resolution at low radio frequencies, to give high sensitivity to faint, filamentary, non-thermal emission. The Canadian Galactic Plane Survey meets these requirements, as has already been demonstrated by the recent discoveries of three faint supernova remnants (Kothes et al. 2001; Kothes 2003).

\section{Observations}

The radio continuum and $\mathrm{HI}$ line data were obtained using the synthesis telescope at the Dominion Radio Astrophysical Observatory (DRAO, Landecker et al. 2000), a seven element east-west interferometer with a maximum baseline of $617 \mathrm{~m}$, as part of the CGPS (Taylor et al. 2003). Single-antenna data are incorporated into the synthesis maps to ensure accurate representation of all structures from the resolution limit up to the largest scales. The low spatial frequency $\mathrm{HI}$ data are from the Low Resolution DRAO Surveys of the CGPS region 
Table 1. Resolution parameters of the observations. The position angle of the beam is defined clockwise from north.

\begin{tabular}{l|cc}
\hline \hline Parameter & G96.0+2.0 & G113.0+0.2 \\
\hline Beam at $408 \mathrm{MHz}$ & $3.5 \times 2.8$ & $3.1 \times 2.8$ \\
Beam at $1420 \mathrm{MHz}$ continuum & $61^{\prime \prime} \times 50^{\prime \prime}$ & $55^{\prime \prime} \times 49^{\prime \prime}$ \\
Beam at $1420 \mathrm{MHz}$ H l line & $72^{\prime \prime} \times 60^{\prime \prime}$ & $66^{\prime \prime} \times 59^{\prime \prime}$ \\
Position Angle & $-47^{\circ}$ & $-72^{\circ}$ \\
CO line & $45^{\prime \prime}$ & $45^{\prime \prime}$ \\
\hline
\end{tabular}

observed with the DRAO 26-m Telescope (Higgs \& Tapping 2000; Higgs et al. 2005). Continuum data are derived from the 408-MHz All-Sky survey (Haslam et al. 1982), and from the $1.4 \mathrm{GHz}$ Effelsberg survey (Reich et al. 1997). The angular resolution of the data varies slightly across the final maps as $\operatorname{cosec}(\delta)$. Resolution parameters are listed in Table 1.

At $1420 \mathrm{MHz}$ the CGPS provides maps for all four Stokes parameters $I, Q, U$, and $V$. The DRAO Synthesis Telescope receives signals in both hands of circular polarization in four separate bands $7.5 \mathrm{MHz}$ wide. The central frequencies of the four bands are 1406.9 MHz, 1414.4 MHz, 1426.9 MHz, and 1434.4 MHz, avoiding the band around the Hi line. Singleantenna data for Stokes $Q, U$, and $V$ are currently not available.

The molecular material was observed in the ${ }^{12} \mathrm{CO}$ line at the Five Colleges Radio Astronomy Observatory (FCRAO) as part of the Outer Galaxy Survey (Heyer et al. 1998), a survey complementary to the CGPS.

\section{Results}

\subsection{Radio continuum emission}

\subsubsection{G96.0+2.0}

Radio continuum maps of the newly discovered extended radio source G96.0+2.0 at $1420 \mathrm{MHz}$ and $408 \mathrm{MHz}$ are shown in Fig. 1. In the $1420 \mathrm{MHz}$ image point sources have been removed by subtracting Gaussians fit to them and the resulting map has been convolved to a resolution of 1.5 to increase the signal to noise ratio. A twisted plane derived from a least square fit to all data points outside the source below $7 \mathrm{~K}$ brightness temperature, which is slightly above the background level in this area, has been subtracted. Since this twisted plane is linear along any cut parallel to the $X$ and $Y$-axis it does not change any structures in the map and is not sensitive to the exact size of the target source. It just removes a background gradient. This was only done for the presentation in Fig. 1. For any analysis the original maps were used.

At both frequencies G96.0+2.0 displays an arc-like structure with a relatively brighter shell to the south-west and smooth emission gradually fading away towards the northeast. The diameter of the visible part is about $26^{\prime}$ as indicated by the circle in Fig. 1 taking the shell as an outer boundary. However, the location of the edge in the north-eastern direction is uncertain. This size together with its appearance implies that G96.0+2.0 is a Galactic object, either a supernova remnant or an H II region. At $1420 \mathrm{MHz}$ the source seems to be located on top of diffuse emission. The nature of the diffuse emission is unknown, but it is most likely of thermal origin since it seems to be brighter relative to G96.0+2.0 at $1420 \mathrm{MHz}$ than at $408 \mathrm{MHz}$. However, parts of it are visible at $408 \mathrm{MHz}$ as well, which makes it very difficult to separate G96.0+2.0 from its background. To get more information about the spectral characteristics we derived radial profiles of the source averaged over the visible position angle range of the shell. We should note at this point that this is of course not a true representation of the actual radial emission profile since this is a 3-dimensional object and each pixel contains all the emission integrated along the line of sight including the approaching and receding parts of the remnant. To calculate these profiles the $1420 \mathrm{MHz}$ image was convolved to the resolution of the $408 \mathrm{MHz}$ observations. We adopted $\ell=96.05^{\circ}$ and $b=1.95^{\circ}$, determined from the circle in Fig. 1, as the centre position of G96.0+2.0. We averaged the radial profiles over the angular range from $90^{\circ}$ to $200^{\circ}$ west of north with rings of width $1^{\prime}$. This covers the visible part of the shell and avoids the bright prominent extragalactic point source visible at the northern tip of the shell at $408 \mathrm{MHz}$. At $1420 \mathrm{MHz}$ this source was visible as well but has been removed from the displayed map. Since the $408 \mathrm{MHz}$ beam is much larger we left the point sources in the data to avoid creating artifacts, due to an uneven background for the Gaussian fit. This is not a problem at $1420 \mathrm{MHz}$ since there the beam is small compared to the SNR candidate.

To subtract the background we linearly fitted between the inner two rings and the next four rings beyond $15^{\prime}$. The final radial profiles are displayed in Fig. 2. The error bars include the r.m.s. noise in the maps and the calibration uncertainties given by Taylor et al. (2003).

The profiles show a slow rise starting from the centre with a sharp outer edge - the decline is comparable to the HPBW of the resolution - typical for a shell-type supernova remnant. The spectral indices $\alpha$ calculated for the individual rings are all between -0.6 and $-0.7\left(S \sim v^{\alpha}\right)$. The integrated flux densities under the radial profiles are $81 \pm 5 \mathrm{mJy}$ and $185 \pm 20 \mathrm{mJy}$ at $1420 \mathrm{MHz}$ and $408 \mathrm{MHz}$, respectively. This results in a spectral index of $\alpha=-0.66 \pm 0.1$.

To get a more reliable value for the spectral index we calculated so-called TT-Plots for G96.0+2.0 by plotting the radial profiles at $1420 \mathrm{MHz}$ and $408 \mathrm{MHz}$ against each other and fitting the slope. We did this for the $408 \mathrm{MHz}$ flux values as a function of the $1420 \mathrm{MHz}$ fluxes and vice versa. The results are plotted in Fig. 3 and confirm the spectral index derived by flux integration.

A spectral index of $\alpha=-0.66$ indicates a non-thermal emission spectrum - for thermal emission of an $\mathrm{H}$ II region we would expect $\alpha=-0.1$. Given its size, its location in the Galactic plane, and its extended diffuse structure, we interpret G96.0+2.0 as a supernova remnant. The radio spectrum is quite steep for a typical mature shell-type supernova remnant for which a spectral index of -0.5 is expected.

Using the flux values integrated at 408 and $1420 \mathrm{MHz}$ results in a radio surface brightness at $1 \mathrm{GHz}$ of $\Sigma_{1 \mathrm{GHz}} \approx$ $8 \times 10^{-23} \mathrm{~W} \mathrm{~m}^{-2} \mathrm{~Hz}^{-1} \mathrm{sr}^{-1}$ for the sector where we did the ring integration. This is one of the lowest radio surface brightnesses known for an SNR.

We did not detect G96.0+2.0 in our polarization data, which means that in the brighter part of the shell the percentage 


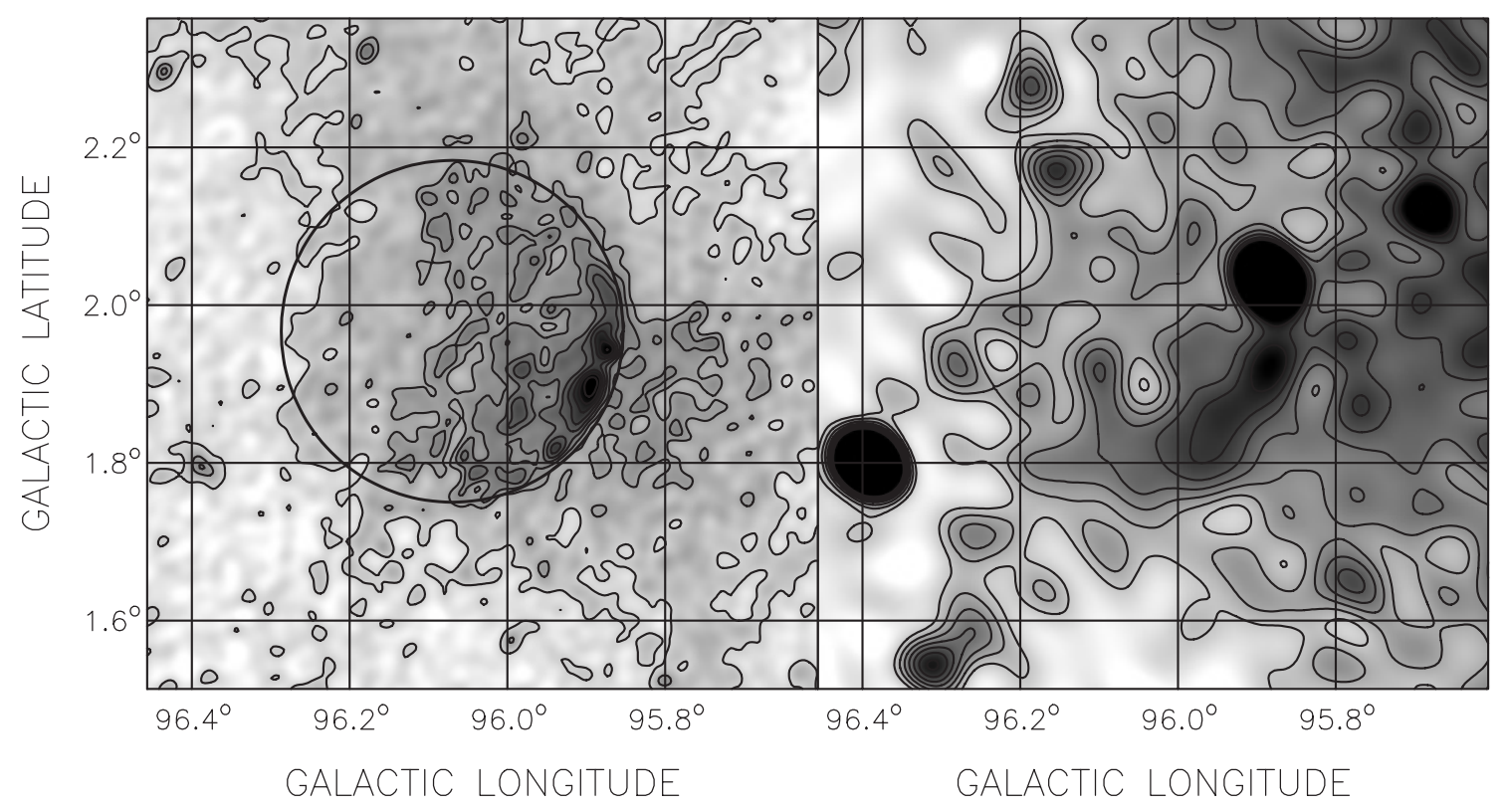

Fig. 1. Radio Continuum maps in Stokes $I$ at $1420 \mathrm{MHz}$ (left) and $408 \mathrm{MHz}$ (right) of G96.0+2.0 taken from the Canadian Galactic Plane Survey. Point sources have been removed from the $1420 \mathrm{MHz}$ image and it has been convolved to 1'.5. Contour levels are at $0.1,0.2,0.25,0.3$, $0.35,0.4,0.45,0.5$, and $0.55 \mathrm{~K}$ at $1420 \mathrm{MHz}$ and at $68,69,70,71,72,73$, and $74 \mathrm{~K}$ at $408 \mathrm{MHz}$. The black circle in the panel describes the dimension of this SNR.

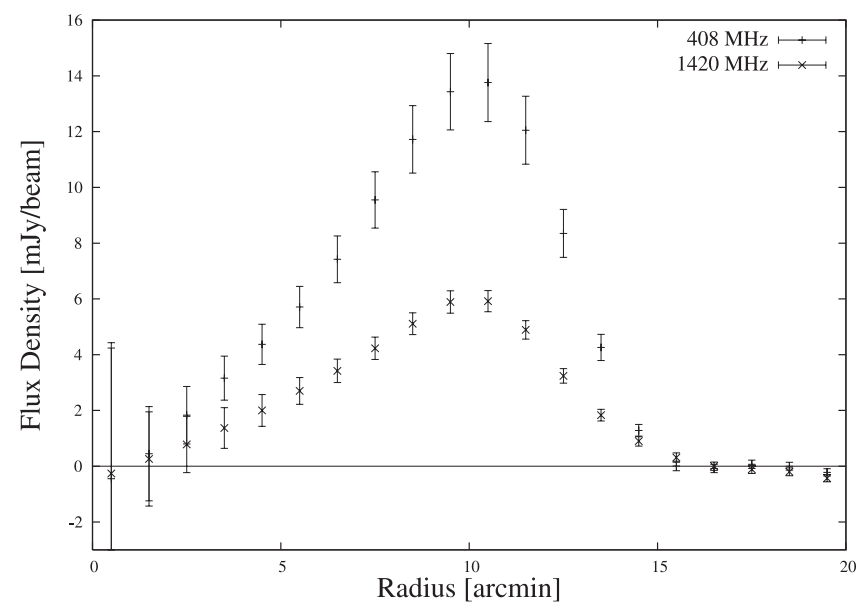

Fig. 2. Radial Profile of G96.0+2.0 at both radio frequencies. The background was fitted linearly between the first two inner rings and the first four rings beyond $15^{\prime}$.

polarization must be well below $15 \%$ assuming that a $3 \sigma$ signal is required for detection. This is not necessarily surprising if the source is rather far away and suffers from beam depolarization due to the foreground magneto-ionic medium.

\subsubsection{G113.0+0.2}

G113.0+0.2 appears to be much brighter than the previously discussed SNR candidate G96.0+2.0. This extended radio source has lain undiscovered because of its proximity to the extremely radio-bright SNR Cassiopeia A, which is located only about 2.5 away. For this reason we do not detect this source in our $408 \mathrm{MHz}$ data. However, since the radio continuum emission from G113.0+0.2 is partly polarized in our $1420 \mathrm{MHz}$

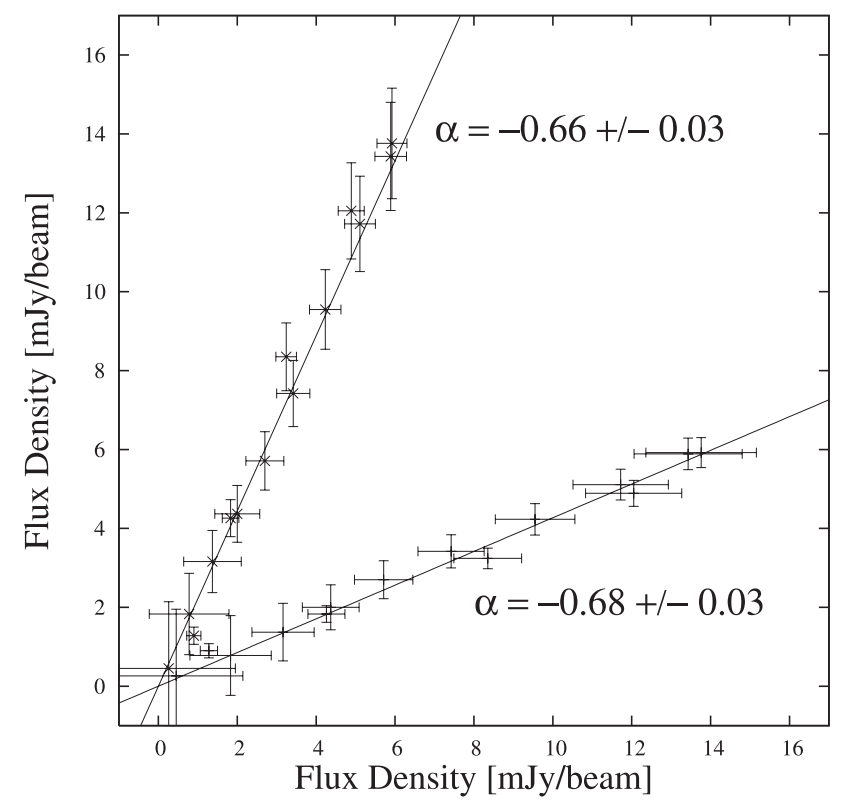

Fig. 3. TT-plots between the radial profiles at $1420 \mathrm{MHz}$ and $408 \mathrm{MHz}$ of G96.0+2.0. The upper result was fitted with the flux at $408 \mathrm{MHz}$ as a function of $1420 \mathrm{MHz}$ and the lower result was fitted at $1420 \mathrm{MHz}$ as a function of $408 \mathrm{MHz}$.

data, we identify it as synchrotron emission. We believe that G113.0+0.2 is most likely a Galactic supernova remnant, since its angular dimensions make an extragalactic origin rather unlikely.

Radio continuum maps of this source at $1420 \mathrm{MHz}$ are shown in Fig. 4 in total power (left) and linearly polarized intensity (right). A twisted plane derived from a least square fit to all data points outside the source below $7.5 \mathrm{~K}$ brightness temperature has been subtracted from the total power map. 


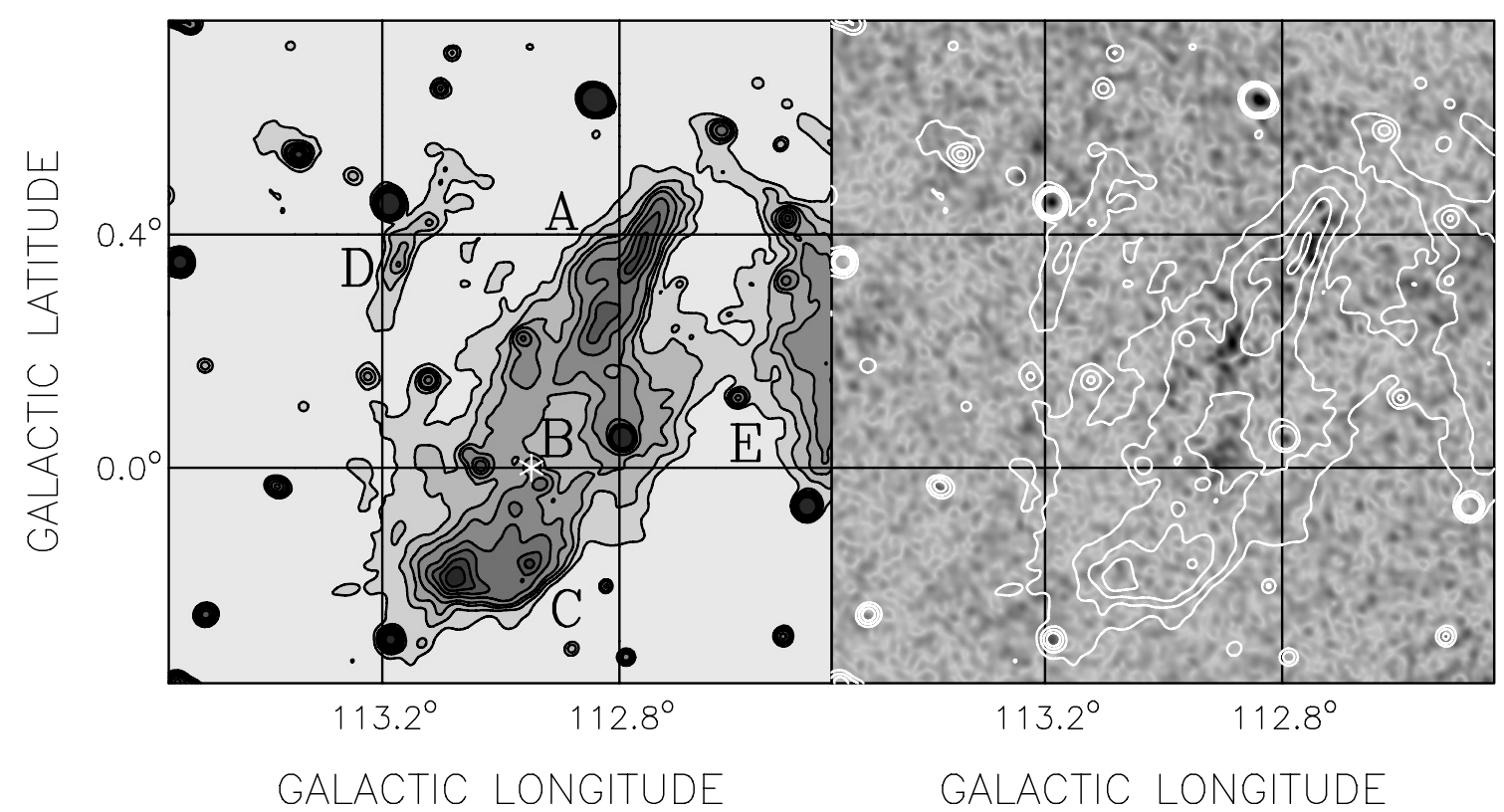

Fig. 4. Radio continuum maps in Stokes I (left) and polarized intensity (right) at $1420 \mathrm{MHz}$ of G113.0+0.2 taken from the Canadian Galactic Plane Survey. The $1420 \mathrm{MHz}$ Stokes $I$ image has been convolved to 1'.5. Contour levels are at 0.15, 0.3, 0.45, 0.6, 0.9, 1.2, 1.5, and 1.8 K for Stokes $I$. For the polarized intensity image we used a linear greyscale between $-0.1 \mathrm{~K}$ (light) and $+0.4 \mathrm{~K}$ (dark). The white contours represent the Stokes $I$ emission. We used every second contour from the left image starting with $0.15 \mathrm{~K}$. The white asterisk marks the position of the pulsar J2326+6113. Different emission regions are labelled A-E (see text).

G113.0+0.2 is located in an area of confusing thermal emission. It consists of a long, linearly polarized filament (Fig. 4: A) which is connected by more diffuse emission (Fig. 4: B) that seems to be polarized, too, to a more compact structure to the south-west (Fig. 4: C). Opposite to the long, straight filament is a smaller fainter arc-like structure (Fig. 4: D) that might be part of the source as well. From our $1420 \mathrm{MHz}$ observations alone we cannot make a final decision on that. To the west of G113.0+0.2 the eastern edge of the H II region Sh 2-162 is also visible in our map (Fig. 4: E). The whole object is about $40^{\prime}$ long and a maximum of $17^{\prime}$ wide, including the faint filament $\mathrm{D}$ with a position angle of $70^{\circ}$ with the Galactic plane.

The CGPS $1420 \mathrm{MHz}$ radio continuum data consists of four bands around $21 \mathrm{~cm}$ wavelength each $7.5 \mathrm{MHz}$ wide. This gives us the opportunity to calculate rotation measure (RM) values for nearby point sources and those parts of G113.0+0.2 which have a sufficient signal to noise ratio in the individual bands. In Fig. 5 we display the polarized intensity map with total power contours and a few average RM values for four bright point sources and a few bright patches from the source itself. The RM values calculated for the emission of G113.0+0.2 range from $-100 \mathrm{rad} / \mathrm{m}^{2}$ in the interior to more than $-300 \mathrm{rad} / \mathrm{m}^{2}$ at the outside edge of the long bright filament. A higher rotation measure at the edge of a supernova remnant would be expected since we are looking along the shell of the SNR there while the angle between the line of sight and the shell is more perpendicular in the interior. It is, however, also possible that the complex structure of the SNR candidates's environment and the area between the source and us causes strong fluctuations in the rotation measure on rather small scales. The wide range of RMs seen for the compact extragalactic sources seems to indicate that. It is quite remarkable that G113.0+0.2 is not completely

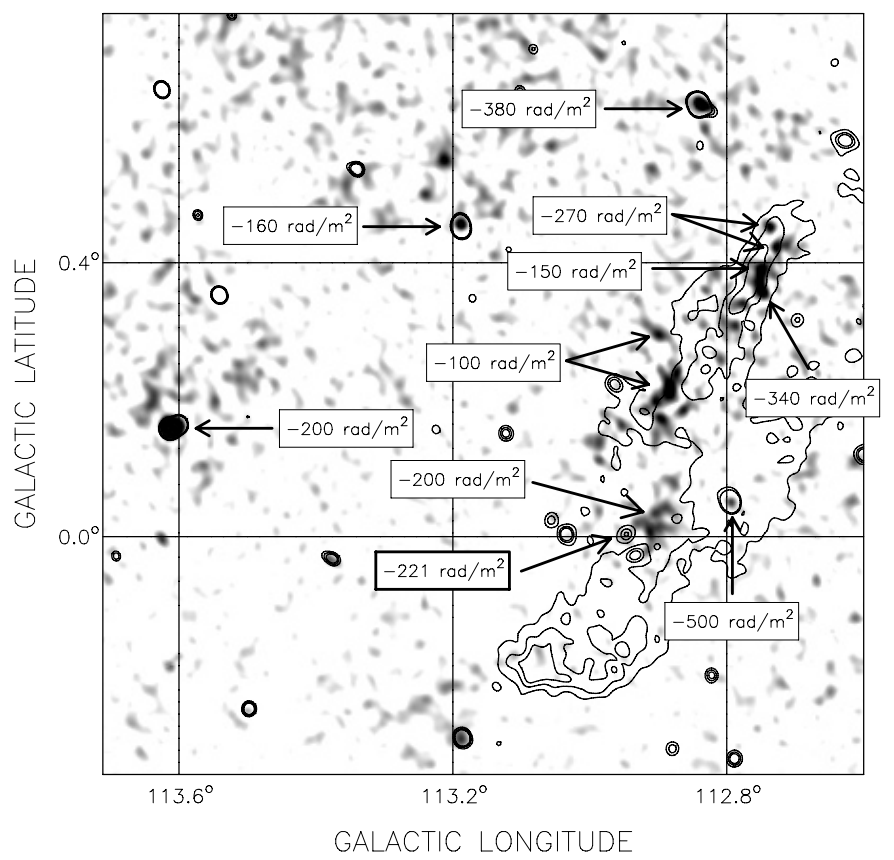

Fig. 5. Map of polarized intensity at $1420 \mathrm{MHz}$ taken from the CGPS. Black contours indicate the total power emission. Values for the rotation measure of 4 point sources and in some areas of the SNR determined with the four bands of the CGPS polarization data are indicated. The rotation measure of $-221 \mathrm{rad} / \mathrm{m}^{2}$ for the pulsar $\mathrm{J} 2326+6113$ was taken from Hamilton \& Lyne (1987).

beam depolarized even though a rotation measure of merely $\pm 36 \mathrm{rad} / \mathrm{m}^{2}$ is enough to rotate the polarization angle at $21 \mathrm{~cm}$ wavelength by $\pm \frac{\pi}{2}$ or $\pm 90^{\circ}$. The magnetic field, responsible for 


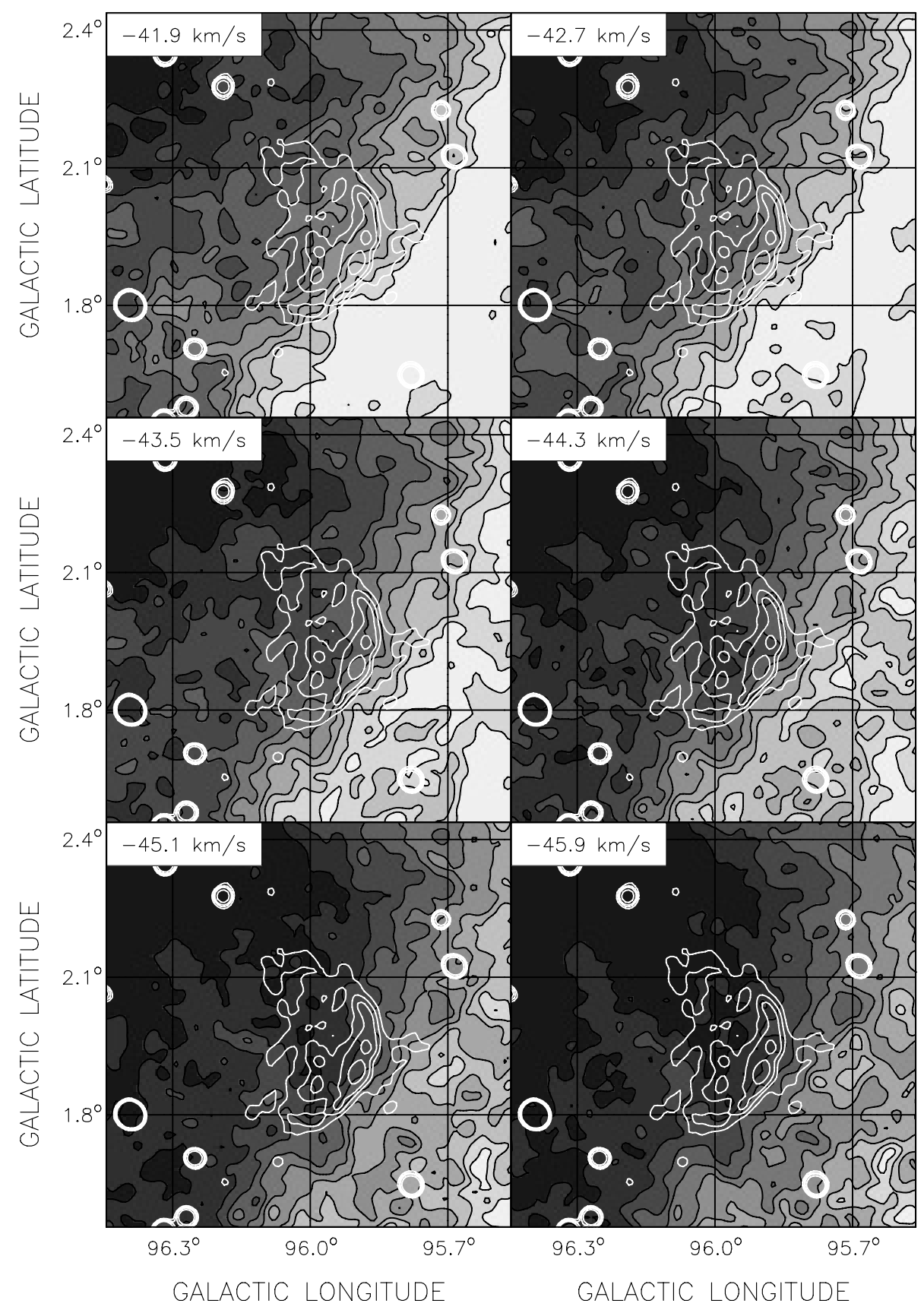

Fig. 6. H I channel maps towards G96.0+2.0 taken from the Canadian Galactic Plane Survey. The H I contours (black) are at 18, 23, 28, 33, 38, $43,48,53$, and $58 \mathrm{~K} T_{\mathrm{B}}$. The white contours indicate the total power emission.

the Faraday Rotation must be quite regular so that the RM fluctuations occur only on scales much larger than the synthesized beam of our observations.

It is also interesting to note that the two compact extragalactic sources closest to the polarized emission of G113.0+0.2 have significantly higher rotation measure values than the emission of the SNR candidate itself, which is another indication for a location within our Galaxy rather than beyond it.

\subsection{The cold environment}

The appearance of a supernova remnant strongly depends on the structure and density of its environment. Hence, to seek explanation for the shapes of the two new SNRs we used the $\mathrm{HI}$ and CO data from the CGPS data base to investigate their ambient media. Additionally we will get information about the systemic velocity of those SNRs, which might give us a kinematic distance. Most progenitor stars of supernova explosions are young and massive objects. Many of those stars create huge bubbles around them by strong stellar winds. Their SNRs expand inside these bubbles which should be detectable in H I observations. Other massive stars which do not have sufficiently strong winds should explode near molecular material from which they were formed themselves. We should find them near dense molecular clouds, which can be associated by shape or by the presence of shocked molecular material. 


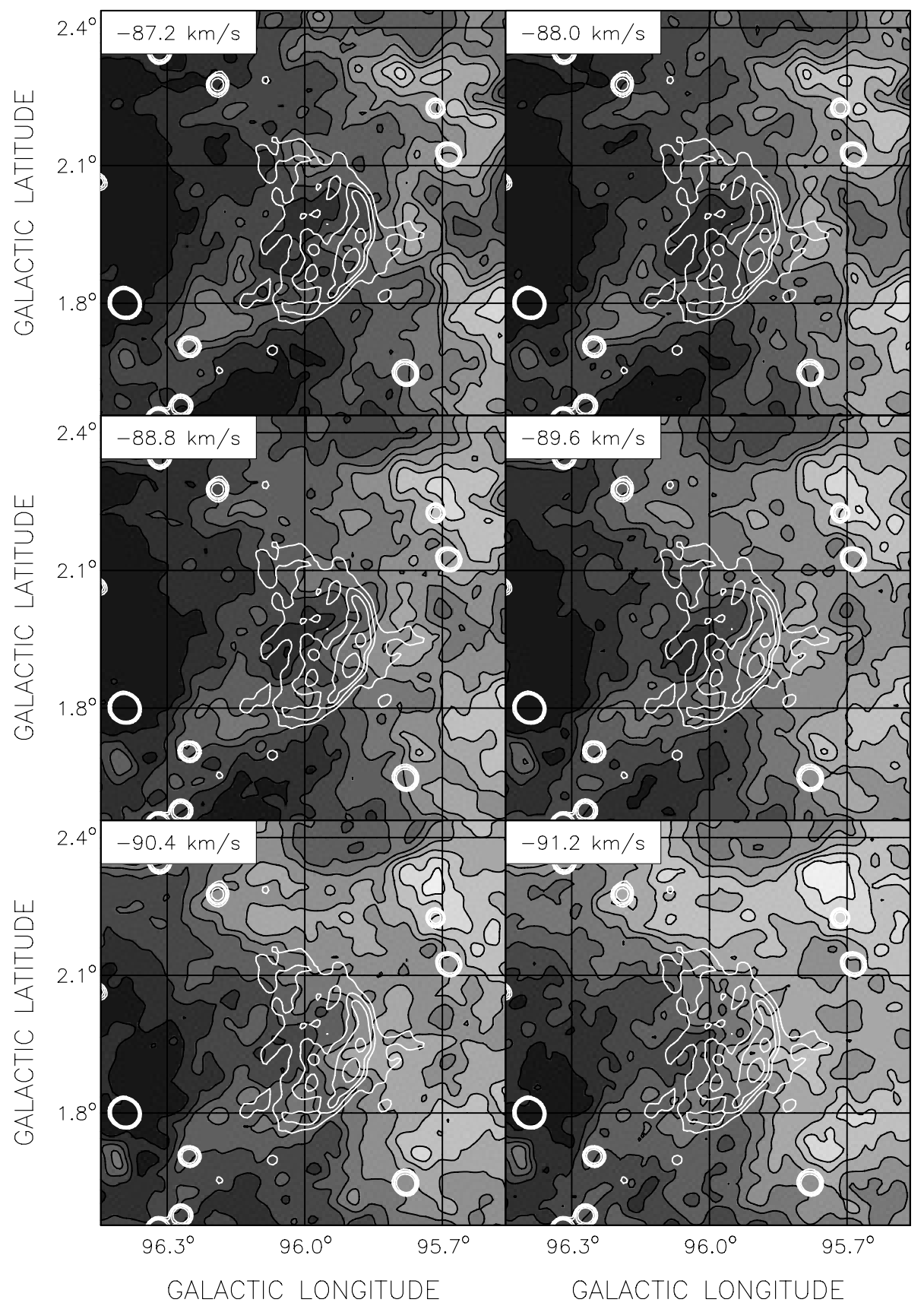

Fig. 7. H I channel maps towards G96.0+2.0 taken from the Canadian Galactic Plane Survey. The H I contours (black) are at 20, 26, 32, 38, 44, $50,56,62$, and $68 \mathrm{~K} T_{\mathrm{B}}$. The white contours indicate the total power emission.

Unfortunately G96.0+2.0 is not covered by the FCRAO Outer Galaxy Survey, so that we have to rely on our H I data alone for this remnant.

\subsubsection{G96.0+2.0}

H I channel maps taken from the Canadian Galactic Plane Survey towards G96.0+2.0 are displayed in Figs. 6 and 7. While investigating the $\mathrm{HI}$ dataset towards this remnant we found two separate structures at two different velocity intervals that might be associated with this SNR. The first structure has a systemic velocity of about $-44 \mathrm{~km} \mathrm{~s}^{-1}$ (see Fig. 6). This would place G96.0+2.0 in the Perseus arm, which is a quite reasonable assumption in this direction of our Galaxy. The SNR would be located at the western edge of a large H I cavity (see Fig. 8), probably a stellar wind bubble created by its progenitor and maybe a few other stars some of which may have not exploded yet and others may even have contributed with their explosion to the shape of this bubble. In this case G96.0+2.0 or its progenitor star would have produced a dent into the western wall of the bubble. The radio continuum emission seems to be located in an area of missing HI. From the centre of the shell to the north the H I follows nicely the curvature of G96.0+2.0 outside the shell. To the south the H I contours look more straight with a gradient perpendicular to the shell. These structures are most pronounced in 
the $-43.5 \mathrm{~km} \mathrm{~s}^{-1}$ channel in Fig. 6. To the east the SNR would expand into the interior of this bubble, which explains its diffuse emission in that direction.

Another possibility appears in the $\mathrm{HI}$ data at a velocity of about $-89 \mathrm{~km} \mathrm{~s}^{-1}$ (see Fig. 7), which would place the SNR in the outer arm, which is a continuation of the Cygnus arm in this direction of our Galaxy. Again, there seems to be an H I structure surrounding the brighter shell of the remnant most pronounced in the velocity channels at $-88.8 \mathrm{~km} \mathrm{~s}^{-1}$ and $-89.6 \mathrm{~km} \mathrm{~s}^{-1}$. This structure has an opening to the east to explain the diffuse emission in that direction, too. In this case, however, the $\mathrm{HI}$ filament does not seem to fit as nicely as the one at $-44 \mathrm{~km} \mathrm{~s}^{-1}$ does, since it overlaps partly with the SNR's radio continuum emission. Additionally due to the large distance to the outer arm, which is about twice as far as Perseus arm in this direction, we consider the Perseus arm location for the SNR more credible. Taking the uncertainty of our estimate and turbulent motions into account we propose a systemic velocity of $-44 \pm 4 \mathrm{~km} \mathrm{~s}^{-1}$.

\subsection{2. $\mathrm{G} 113.0+0.2$}

For the new SNR candidate G113.0+0.2 the full CGPS data set including the ${ }^{12} \mathrm{CO}(1-0)$ line measurements is available. Channel maps for the $\mathrm{CO}$ and $\mathrm{H}$ I observations are displayed in Figs. 9 and 10, respectively. For the CO channel maps we display a smaller area around the bright head of the source to emphasize the structural coincidence of the radio continuum emission and the distribution of the $\mathrm{CO}$ clouds. Going from lower to higher negative velocities at $-49.3 \mathrm{~km} \mathrm{~s}^{-1}$ the $\mathrm{CO}$ emission appears and seems to be wrapped around the south-eastern edge of the bright radio emission. At $-50.1 \mathrm{~km} \mathrm{~s}^{-1}$ two bright molecular clouds with a narrow valley between them - which marks the location of the radio continuum emission - appear, remain until $-50.9 \mathrm{~km} \mathrm{~s}^{-1}$ and then fade away at higher negative velocities. This is a striking coincidence and gives G113.0+0.2 a very credible systemic velocity of about $-50 \mathrm{~km} \mathrm{~s}^{-1}$. The same velocity channels in H I are shown in Fig. 10. Here we display a field of view of about $2^{\circ}$ to get a better look at the full environment of this supernova remnant candidate.

G113.0+0.2 seems to be located inside a huge butterflyshaped cavity in the HI distribution. There is no coincidental H I structure around the bright head of the remnant, which is interacting with the molecular cloud. However, on the right hand side of the long straight filament there appears to be a gradient in the $\mathrm{HI}$ at the $-50.1 \mathrm{~km} \mathrm{~s}^{-1}$ channel with a slope that runs perpendicular to this filament. It prevails in the next channel and then changes shape towards the higher negative velocities. On its own this would hardly be enough evidence for an interaction, but together with the $\mathrm{CO}$, which agrees in shape very well with the structure of the bright head we believe we have enough evidence to propose a systemic velocity of $-50 \pm 3 \mathrm{~km} \mathrm{~s}^{-1}$ for $\mathrm{G} 113.0+0.2$, which would make it a resident of the Perseus arm.

The striking coincidence of the $\mathrm{CO}$ emission with the structure of the radio continuum emission together with the evidence

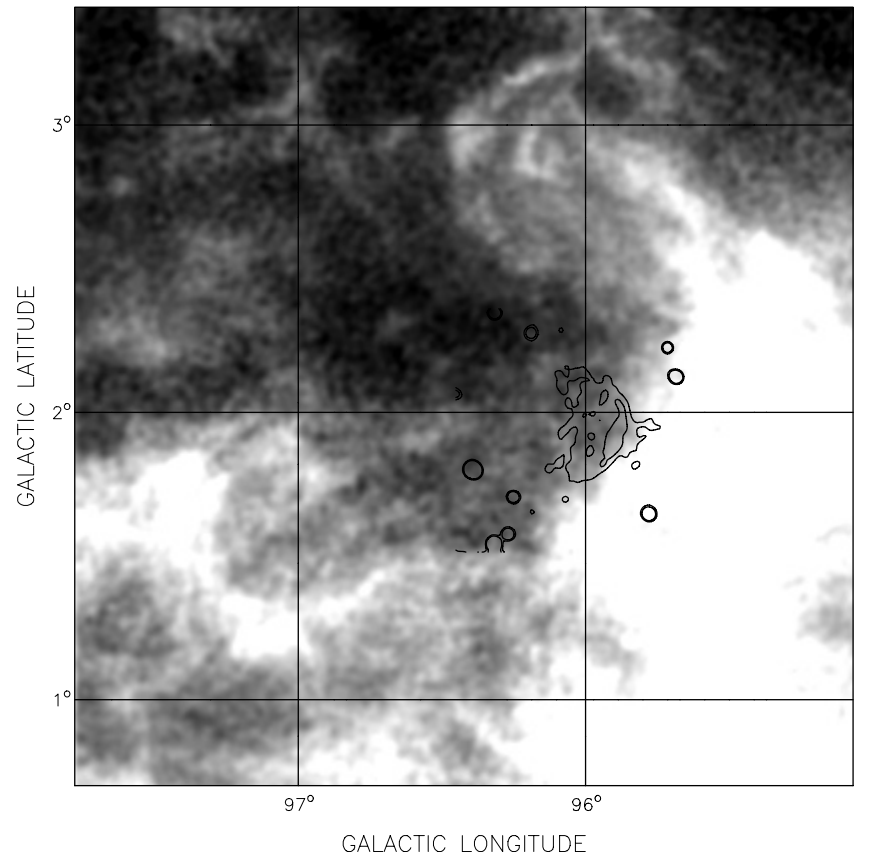

Fig. 8. H I channel map at $-43.5 \mathrm{~km} \mathrm{~s}^{-1}$ towards G96.0+2.0 taken from the Canadian Galactic Plane Survey with a much larger field of view than in Figs. 6 and 7. The grayscale is linearly plotted from $10 \mathrm{~K}$ (black) to $50 \mathrm{~K}$. The black contours indicate the total power emission.

presented in Sect. 3.1.2 makes an interpretation of G113.0+0.2 as a Galactic supernova remnant almost unavoidable.

\section{Discussion}

\subsection{G96.0+2.0}

A systemic velocity of $-44 \mathrm{~km} \mathrm{~s}^{-1}$ makes G96.0+2.0 a Perseus spiral arm object in this direction of our Galaxy. To determine its distance more precisely we used the method published by Foster \& Routledge (2003) and Foster (2005). This method uses a model for the spatial density and velocity field traced by the distribution of $\mathrm{HI}$ in the outer disk of the Galaxy, to derive a distance-velocity relation that can be used to calculate a distance to any object with a known systemic velocity. For G96.0+2.0 the proposed systemic velocity of $-44 \mathrm{~km} \mathrm{~s}^{-1}$ translates to a distance of $4.0 \mathrm{kpc}$. At this distance the SNR would have a diameter of about $30 \mathrm{pc}$. As indicated by the circle in Fig. 1, the portion of the SNR visible in radio emission is almost circular, implying that it has so far expanded into a homogeneous medium in all directions. Only to the east its dimension cannot be traced by radio emission, probably due to a density gradient into the interior of the $\mathrm{H}$ I bubble. This also means that all the visible shell should be in the same evolutionary stage, most likely still expanding freely, since the radio spectrum is quite steep and the remnant is still very faint.

Fermi acceleration in shocks (Bell 1978a,b; Blandford \& Ostriker 1978) is believed to be the main mechanism producing the relativistic electrons responsible for synchrotron emission. The radio spectral index $\alpha$ in adiabatic shocks is predicted to be: $\alpha=-\frac{3}{2(r-1)}$. Here $r$ is the shock compression ratio. SNRs 


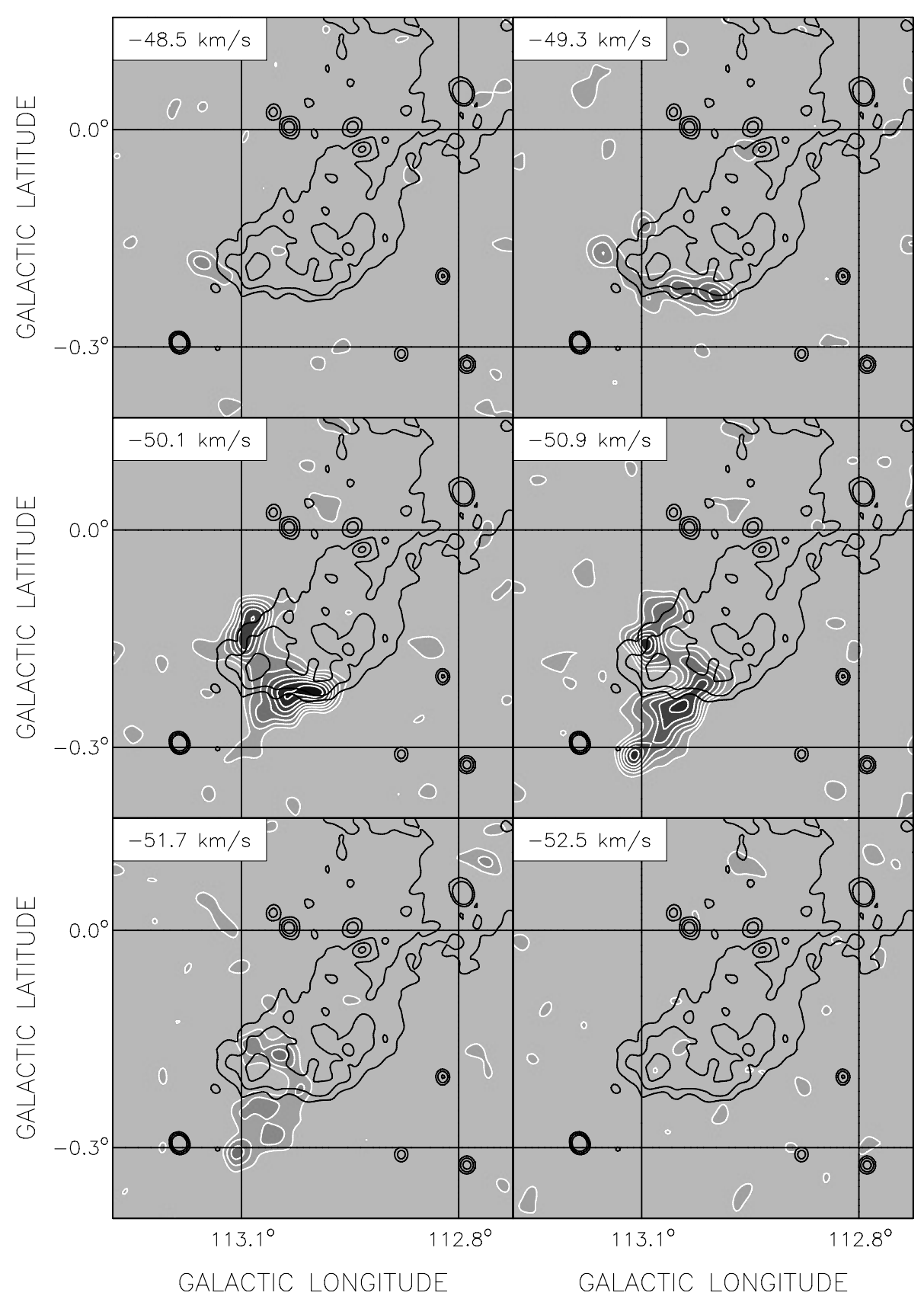

Fig. 9. ${ }^{12} \mathrm{CO}(1-0)$ channel maps towards G113.0+0.2 taken from the Canadian Galactic Plane Survey. The CO contours (white) are at 0.3 , 0.5, $0.7,0.9,1.1,1.3$, and $1.5 \mathrm{~K} T_{\mathrm{B}}$. The black contours indicate the total power emission.

in early stages of their evolution, that is the known historical shell-type SNRs, show steeper radio spectra (Kepler's SNR: $\alpha=-0.64, \mathrm{G} 11.2-0.3: \alpha=-0.6$, Cas A: $\alpha=-0.77$, Tycho's SNR: $\alpha=-0.61$, and SN 1006: $\alpha=-0.6$, see Green 2004a,b) corresponding to low compression ratios between 3 and 3.5. Adiabatically expanding SNRs, SNRs in the so-called Sedov phase, have radio spectra with $\alpha=-0.5$ corresponding to a compression ratio of $r=4$. For older SNRs the compression ratio is increasing further. Thus the slope of the spectrum does not reflect the actual age of the remnant as a number in years but rather the evolutionary stage. Freely expanding SNRs have steep spectra, adiabatically expanding remnants have spectra with $\alpha \approx-0.5$, and when the SNR becomes radiative the spectrum may flatten further. However, a supernova remnant can be several thousand years old and still expand freely if it exploded inside a stellar wind bubble. This could be the case for G96.0+2.0. In the middle of the shell it might just be starting to interact significantly with the H I wall, entering the adiabatic phase of evolution, which explains why the remnant is brightest there.

The huge Hi bubble, in which the SNR is residing, has a diameter of about $100 \mathrm{pc}$ at the distance of $4.0 \mathrm{kpc}$, with an opening to the north-east. The dent in its wall, which surrounds G96.0+2.0 should have been created by the SNR's progenitor, since otherwise the SNR should expand mostly adiabatically now and there should be a change of curvature away 


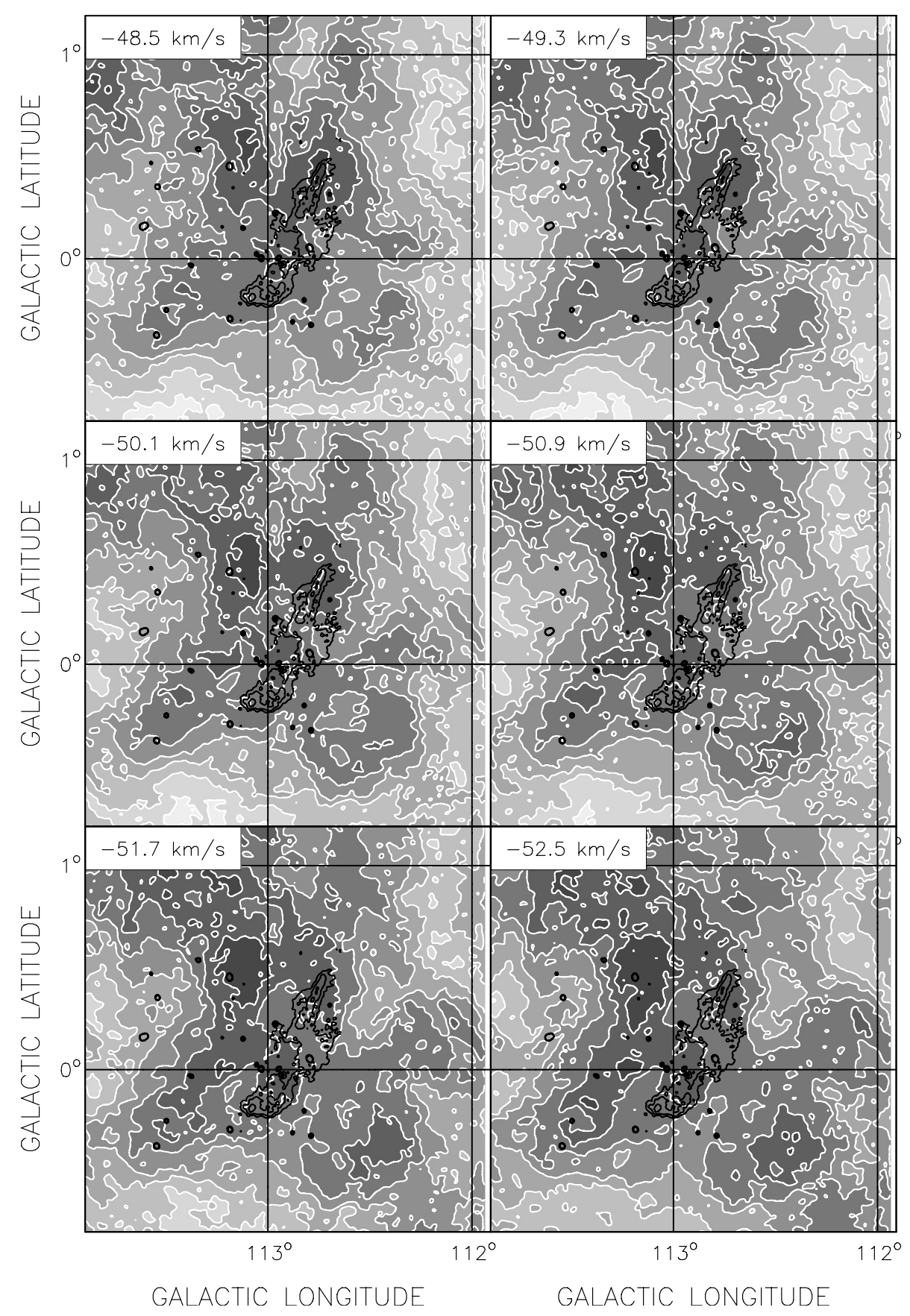

Fig. 10. H I channel maps towards G113.0+0.2 taken from the Canadian Galactic Plane Survey. The H I contours (white) are at 40, 50, 60, 70, 80 , 90 , and $100 \mathrm{~K} T_{\mathrm{B}}$. The black contours indicate the total power emission.

from the brightest part of the shell. The SNR shockwave would hit the wall first in the centre where the shell is brightest, producing a smoother curvature there, which is, as shown with the circle in Fig. 1, evidently not the case. This makes the SNR the remnant of a type Ib/c explosion, since their progenitor stars produce a strong stellar wind. The huge bubble, however, must have been created by the combined effects of a group of massive stars by their winds and maybe earlier supernova explosions. Whether there are still active sources of stellar winds inside this bubble cannot be determined from our current observations.

\section{2. $G 113.0+0.2$}

The shape of G113.0+0.2, with its straight arc-like filament and sharp outer edge in most parts, indicates that it belongs to the group of shell-type supernova remnants. An adiabatically expanding shell-type SNR would have a spectral index around $\alpha=-0.5$, a fact we cannot test with our current observations due to the proximity of Cassiopeia A, which confuses the radio emission at $408 \mathrm{MHz}$. Other useful observations at different frequencies are not available.

Having established the systemic velocity of the molecular cloud and the huge stellar wind bubble we can again use the 
method of Foster \& Routledge (2003) and Foster (2005) to derive a kinematic distance. In the direction of G113.0+0.2 its radial velocity of about $-50 \mathrm{~km} \mathrm{~s}^{-1}$ translates to a distance of $3.1 \mathrm{kpc}$ which would place the SNR at the position of the spiral shock in the Perseus arm. That makes the remnant about $36 \mathrm{pc}$ long and $15 \mathrm{pc}$ wide with an angle of $70^{\circ}$ with the Galactic plane. The host stellar wind bubble is in the middle, at its narrowest, about $40 \mathrm{pc}$ wide and in the south about $80 \mathrm{pc}$.

This huge butterfly-shaped stellar wind bubble in which the SNR resides is most likely the result of combined effects of a group of stars with their winds and possibly enhanced by supernova explosions. We cannot say from our current observations, whether the progenitor of G113.0+0.2 contributed to this structure with its own strong wind. However, the fact that the bright head is closely interacting with a dense molecular cloud indicates a star without a strong wind. In this case the progenitor exploded in a type II event.

Close to the centre of the SNR we found the pulsar B 2324+60 also known as $\mathrm{J} 2326+6113$ at $\ell=112.95^{\circ}$ and $b=0.00^{\circ}$ (Fig. 4). It is even visible in our $1420 \mathrm{MHz}$ data as a compact source of about $6 \mathrm{mJy}$. It has a low period of $234 \mathrm{~ms}$, comparable to pulsar B1643-43 (232 ms) in SNR G341.2+0.9 and pulsar B1853+01 (267 ms) in SNR W 44 (Frail et al. 1994; Wolszczan et al. 1991, respectively), but its characteristic age is $\tau \approx 10^{7} \mathrm{yr}$ (see the ATNF Pulsar Catalogue: http:// www . atnf.csiro.au/research/pulsar/psrcat/). Its dispersion measure of DM $=123 \mathrm{pc} \mathrm{cm}^{-3}$ gives it a distance of $4.8 \mathrm{kpc}$ using the model of free electrons in our Galaxy by Taylor \& Cordes (1993). Mitra et al. (2003) found that the rotation measure of $-221 \mathrm{rad} \mathrm{m}^{-2}$ (Hamilton \& Lyne 1987) is unusually high. These authors argue that the high RM and DM values of B 2324+60 and a few other pulsars nearby, result from enhanced electron densities, indicated by bright $\mathrm{H}_{\alpha}$ emission that is related to the about $3 \mathrm{kpc}$ distant $\mathrm{H}$ II regions Sh 2-161, Sh 2-162, and Sh 2-163. In this case the pulsar B $2324+60$ is significantly closer than its catalogued DM distance of $4.8 \mathrm{kpc}$, but still must be beyond the H II regions that create the enhanced dispersion measure. This would put the pulsar very close to our new SNR G113.0+0.2 and probably inside the butterfly-shaped stellar wind bubble.

It can be seen from Fig. 5 that the rotation measure of the pulsar $\left(-211 \mathrm{rad} / \mathrm{m}^{2}\right)$ is very close to that of the nearby SNR emission ( $\left.-200 \mathrm{rad} / \mathrm{m}^{2}\right)$ suggesting a very similar distance. Also the high RM values found on the SNR seem to indicate some influence of those $\mathrm{H}$ II regions. A compelling explanation would be that all of these structures are part of the same complex at a distance of about $3 \mathrm{kpc}$. The similar systemic velocities seem to confirm this. For the H II regions Sh 2-161b, Sh 2-162, and Sh 2-163 Brand \& Blitz (1993) derived -51.9, -44.7 , and $-44.9 \mathrm{~km} \mathrm{~s}^{-1}$, respectively.

If the pulsar is related to the SNR, it must have been born with an unusually low magnetic field, resulting in a low period derivative, giving it a high initial characteristic age. The shape of the SNR would be difficult to explain since the pulsar is located on a line described by the straight filament, which should have been created by a shock wave coming from the east. If the pulsar and the SNR were born in the same supernova the pulsar must originate from the same location as the shock wave.
This would imply that the pulsar travelled at least $0.3^{\circ}$, more probably $0.4^{\circ}$, perpendicular to the line of sight since it was born. For an age of $20000 \mathrm{yr}$ the pulsar would have had to travel at 800 and $1050 \mathrm{~km} \mathrm{~s}^{-1}$, respectively, which are acceptable pulsar velocities.

A correlation of the pulsar J 2326+6113 with the SNR G113.0+0.2 is quite compelling and, given the evidence presented here, we can at least assume that they are very close to each other. However, a final decision on this association cannot be made at this point. Given its high characteristic age it is more likely that the pulsar was created in an earlier supernova explosion that also contributed to the shape of the stellar wind bubble.

\section{Summary}

Two new Galactic supernova remnants, G96.0+2.0 and G113.0+0.2, have been discovered in the data of the Canadian Galactic Plane Survey. Both sources show characteristics of shell-type SNRs and are located in the Perseus spiral arm at distances of 4.0 and $3.1 \mathrm{kpc}$, respectively. The radio continuum emission of G96.0+2.0 consists of a shell to the west and slowly fades away to the east with no visible edge in this direction. The SNR has a radio spectral index of $\alpha \approx-0.66$ and the visible part of the remnant has a diameter of about $30 \mathrm{pc}$. It is located at the edge of a large stellar wind bubble and is most likely the result of a type $\mathrm{Ib} / \mathrm{c}$ supernova explosion. G113.0+0.2 has an unusual elongated structure and consists of a long, linearly polarized filament and a bright head structure to the south that might be connected by diffuse polarized emission. This newly discovered SNR is about $36 \mathrm{pc}$ long and up to $15 \mathrm{pc}$ wide at an angle of $70^{\circ}$ with the Galactic plane. A correlation with the pulsar $\mathrm{J} 2326+6113$ is quite compelling, but requires a rather high transverse velocity for the pulsar combined with an old age for the SNR. G113.0+0.2 is located in a large butterflyshaped stellar wind bubble and its bright head is interacting with a number of small molecular clouds. The SNR might be part of a huge $\mathrm{H}$ II region and SNR complex that also contains Sh 2-161, Sh 2-162, and Sh 2-163.

Acknowledgements. We wish to thank Tyler Foster for providing the distances to G113.0+0.2 and G96.0+2.0 with his new method. We also wish to thank Tom Landecker for careful reading of the manuscript. The Dominion Radio Astrophysical Observatory is a National Facility operated by the National Research Council. The Canadian Galactic Plane Survey is a Canadian project with international partners, and is supported by the Natural Sciences and Engineering Research Council (NSERC). For our research we made use of the ATNF Pulsar Catalogue, (Web address: http://www.atnf.csiro.au/research/pulsar/psrcat/), provided by the Commonwealth Scientific and Industrial Research Organisation (CSIRO) of Australia.

\section{References}

Bell, A. R. 1978a, MNRAS, 182, 147

Bell, A. R. 1978b, MNRAS, 182, 443

Blandford, R. D., \& Ostriker, J. P. 1978, ApJ, 221, L29

Brand, J., \& Blitz, L. 1993, A\&A, 275, 67 
Foster, T., \& Routledge, D. 2003, ApJ, 598, 1005

Foster, T. 2005, ApJ, submitted

Frail, D. A., Goss, W. M., \& Whiteoak, J. B. Z. 1994, ApJ, 437, 781

Green, D. A. 2004a, A Catalogue of Galactic Supernova Remnants (2001 December version), Mullard Radio Astronomy Observatory, Cavendish Laboratory, Cambridge, UK (available on the WorldWide-Web at http://www.mrao.cam.ac.uk/surveys/snrs/) Green, D. A. 2004b, Bull. Astron. Soc. Ind., 32, 335

Hamilton, P. A., \& Lyne, A. G. 1987, MNRAS, 224, 1073

Haslam, C. G. T., Stoffel, H., Salter, C. J., \& Wilson, W. E. 1982, A\&AS, 47, 1

Heyer, M. H., Brunt, C., Snell, R. L., et al. 1998, ApJS, 115, 241

Higgs, L. A., \& Tapping, K. F. 2000, AJ, 120, 2471
Higgs, L. A., Landecker, T. L., Asgekar, A., et al. 2005, AJ, 129, 2750

Kothes, R., Landecker, T. L., Foster, T., \& Leahy, D. A. 2001, A\&A, 376, 641

Kothes, R. 2003, A\&A, 408, 187

Landecker, T. L., Dewdney, P. E., Burgess, T. A., et al. 2000, A\&AS, 145,509

Mitra, D., Wielebinski, R., Kramer, M., \& Jessner, A. 2003, A\&A, 398, 993

Reich, P., Reich, W., \& Fürst, E. 1997, A\&AS, 126, 413

Taylor, J. H., \& Cordes, J. M. 1993, ApJ, 411, 674

Taylor, A. R., Gibson, S. J., Peracaula, M., et al. 2003, AJ, 125, 3145

Wolszczan, A., Cordes, J. M., \& Dewey, R. J. 1991, ApJ, 372, L99 